\title{
Search for BSM physics with neutron beta decay in the BRAND project
}

\author{
K. Bodek, ${ }^{a}$ J. Choi, ${ }^{b}$ L. De Keukeleere, ${ }^{c}$ K. Dhanmeher, ${ }^{d}$ M. Engler, ${ }^{e}$ G. Gupta, ${ }^{a}$ \\ A. Kozela, ${ }^{d}$ K. Lojek, ${ }^{a}$ K. Pysz, ${ }^{d}$ D. Ries, ${ }^{e}$ D. Rozpedzik, ${ }^{a, *}$ N. Severijns, ${ }^{c}$ T. Soldner,$~^{f}$ \\ N. Yazdandoost, ${ }^{e}$ A. R. Young ${ }^{b}$ and J. Zejma ${ }^{a}$ \\ ${ }^{a}$ Institute of Physics, Jagiellonian University, Krakow, Poland \\ ${ }^{b}$ Department of Physics and Astronomy, NC State University, Raleigh, USA \\ ${ }^{c}$ Institute of Nuclear and Radiation Physics, KU Leuven, Belgium \\ ${ }^{d}$ Institute of Nuclear Physics, Polish Academy of Sciences, Krakow, Poland \\ e Johannes Gutenberg University Mainz, Germany \\ ${ }^{f}$ Institut Laue-Langevin, Grenoble, France \\ E-mail: dagmara.rozpedzik@uj.edu.pl
}

\begin{abstract}
Neutron and nuclear beta decay correlation coefficients are sensitive to the exotic scalar and tensor interactions hypotheses that are beyond the Standard Model (BSM). The BRAND project aims at a test of the Lorentz structure of weak interaction in neutron decay by precision measurements of yet unexplored transverse polarization of electrons in correlation with the neutron spin and electron and recoil proton momenta. The experiment will simultaneously measure eleven neutron correlation coefficients ( $a, A, B, D, H, L, N, R, S, U, V)$, where seven of them $(H, L, N, R, S, U$ and $V$ ) depend on the transverse electron polarization. Five of these correlations: $H, L, S, U$ and $V$ were never attempted experimentally before. The expected ultimate sensitivity of the proposed experiment with respect to BSM couplings will be comparable to that of the ongoing and planned correlation measurements in neutron and nuclear beta decays but offers completely different systematics and additional sensitivity to imaginary parts of the scalar and tensor couplings. In the presentation, an overview of the project, physical motivation and applied experimental techniques were reported. The results of the first pilot run of the experiment performed recently using the cold neutron beam line PF1B at the Laue-Langevin Institute, Grenoble, France were presented, with an emphasis on the challenges of the proposed proton detection technique.
\end{abstract}

\footnotetext{
*** 22nd Particles and Nuclei International Conference ***

*** 05-10 September $2021 * * *$

*** Lisbon, Portugal ***
}

\footnotetext{
${ }^{*}$ Speaker
} 


\section{Physics motivation}

The proposed experiment will simultaneously measure eleven neutron correlation coefficients, where seven of them $(H, L, N, R, S, U$ and $V)$ depend on the transverse electron polarization. Non zero values of these angular correlations would signal nonexistent in the Standard Model (SM) but allowed by the Lorentz structure exotic contributions to the weak interactions provided the electromagnetic corrections are properly taken into account. A measurement of these coefficients with the absolute accuracy of $5 \times 10^{-4}$ will allow for significantly improved constraints on real and imaginary parts of the scalar and tensor terms leading to new bounds on the leptoquark exchange model, R-parity violating MSSM and EFT parameters [1].

\section{Experimental methods and test setup}

The experimental techniques applied in the BRAND project are different from that used in the past and in the ongoing neutron decay correlation experiments, so different are also the associated systematic effects [1]. The strategy of the proposed experiment is to reconstruct event-by-event the decay kinematics and identify the decay origin which must be localized in the beam fiducial volume. Fixing the three-body decay kinematics by the measured electron energy and relative e-p angle one realizes that the proton energy and thus the proton time-of-flight must choose between two discrete and known values. The measurement of momenta of electrons and protons in coincidence allows for reconstruction of the antineutrino momentum and provides access to the terms dependent on this quantity. The proposed ultimate experimental setup will profit from efficient cylindrical detector geometry with electron tracking in a multi-wire drift chamber (MWDC) with signal readout at both wire ends based on the miniBETA spectrometer technique [2,3], detection of both direct and Mott-scattered electrons in plastic scintillator hodoscopes as well as conversion of accelerated protons into bunches of electrons and their subsequent detection in a thin plastic scintillator [4]. The advanced polarimetry techniques such as periodic neutron spin flip during data taking and evaluation of asymmetry and super-ratio in data analysis will be applied for the reduction of
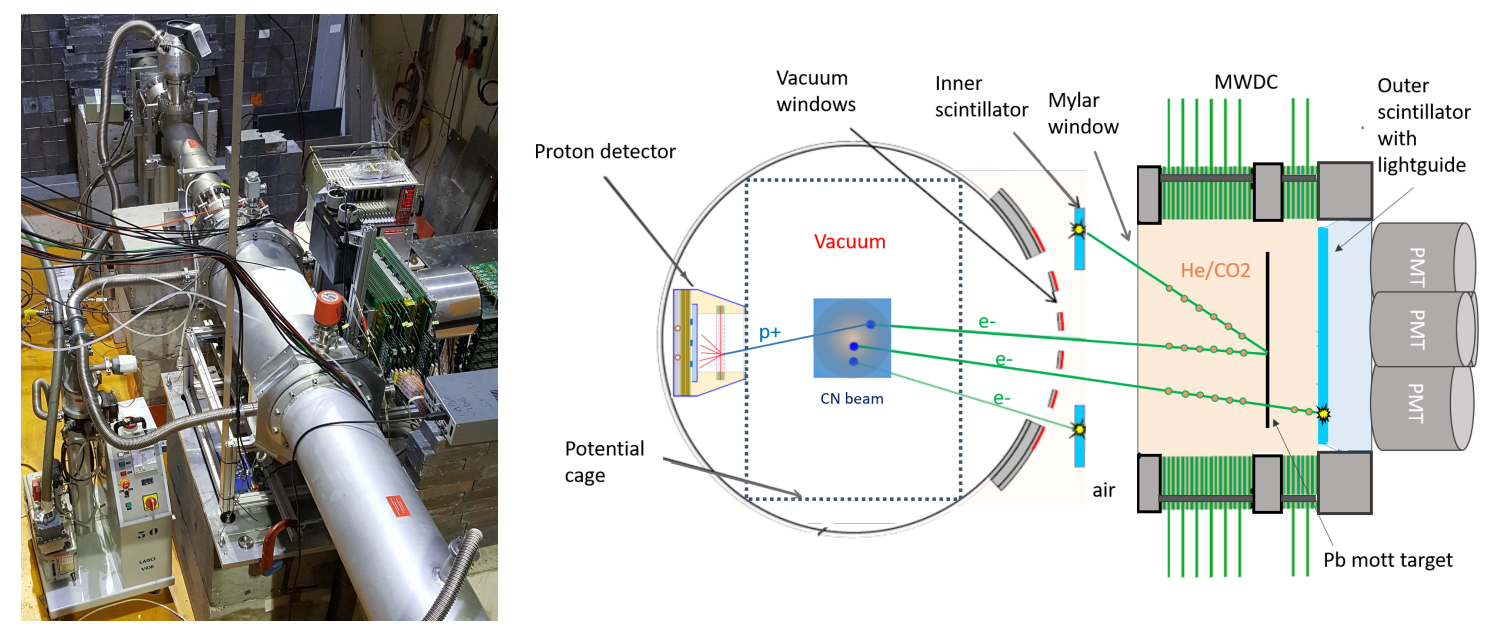

Figure 1: Experiment layout of the test setup at ILL, September 2020. 


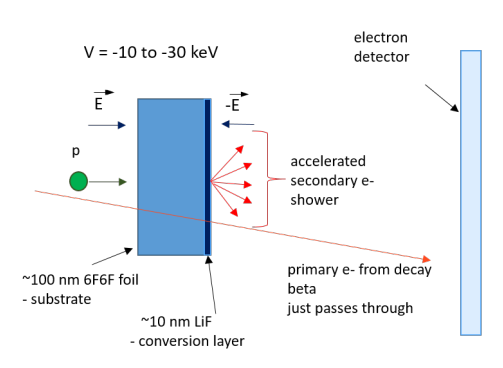

a)
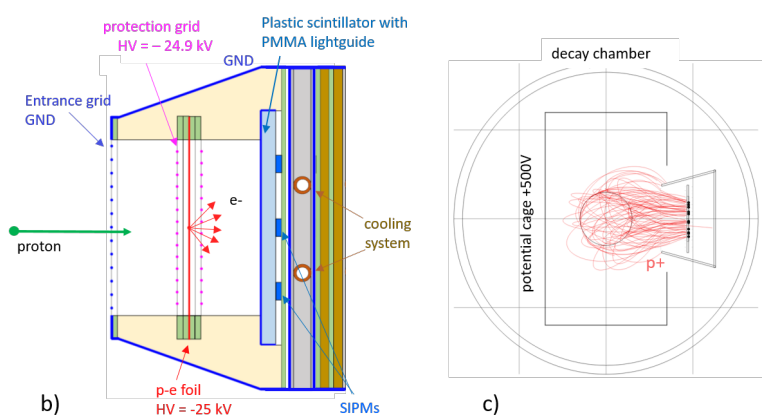

c)

Figure 2: Proton detection technique: a) Experimental method [4] b) Detector prototype scheme c) COMSOL simulation of proton transport.

geometry misalignment related systematic effects [1]. The prototypes of detectors were tested using the cold-neutron beam. A layout of the test setup is presented in Figure 1.

\section{Proton detection technique and detector prototype design}

In the BRAND project, the proton detection should meet several requirements. It is crucial that the detector is able to distinguish between recoiling protons and decay electrons - which means: between electron bunches consisting of several low energy (approx. $25 \mathrm{keV}$ ) electrons and single higher energy ( $>150 \mathrm{keV}$ ) decay electrons. Moreover, the detectors must be made of low $Z$ materials in order to minimize the backscattering effects. Therefore the proton detection technique developed at NCSU by S. Hoedl et al. [4] has been chosen. In this method the recoil protons are accelerated in the external electric field to about -10 to $-30 \mathrm{keV}$ and converted in a $\mathrm{LiF}$ thin layer into bunches of electrons. Next, electrons are accelerated and detected by a plastic scintillation detector according the the scheme shown in Fig. 2a. In the asymmetric electric field barrier, decay electrons would experience not only energy change but also they would acquire additional phase between momentum and spin vectors. This effect is substantial therefore the symmetric barrier is planned in BRAND such that the deflection of the electron momentum is compensated. The remnant effects due to possible local non-uniformities of the electric field will be further suppressed by symmetry of the detector arrangement: the numbers of events with negative and positive deflection angle will be about equal. The second key point in proton detection is the choice of a proper secondary electron (electron bunches) detector. Such a detector must be fast enough as it will participate in the time-of-flight measurement with the resolution of a few nanoseconds and must be position sensitive with the resolution of about $10 \mathrm{~mm}$ to allow for reconstruction of proton energy in the decay origin. It is equally important that the detector should be robust to the high electric field and insensitive to the neutron spin guiding magnetic field. The proton detector prototype proposed and developed at JU fulfills all these requirements. As the first choice we proposed a thin $(25 \mu \mathrm{m})$ plastic scintillator layer deposited on a PMMA lightguide. The scintillation light will be detected by a set of SiPM sensors $\left(6 \times 6 \mathrm{~mm}^{2}\right)$ arranged on a triangular mesh attached to the PMMA lightguide plate. The hit position is deduced from the light distribution on the neighbouring sensors close to the scintillation light origin. The best resolution is obtained by calculating the centroid with weights taken as squares of the light intensity registered by the SiPMs. The cross section of the proton detector prototype is presented in Fig. 2b. The detector 

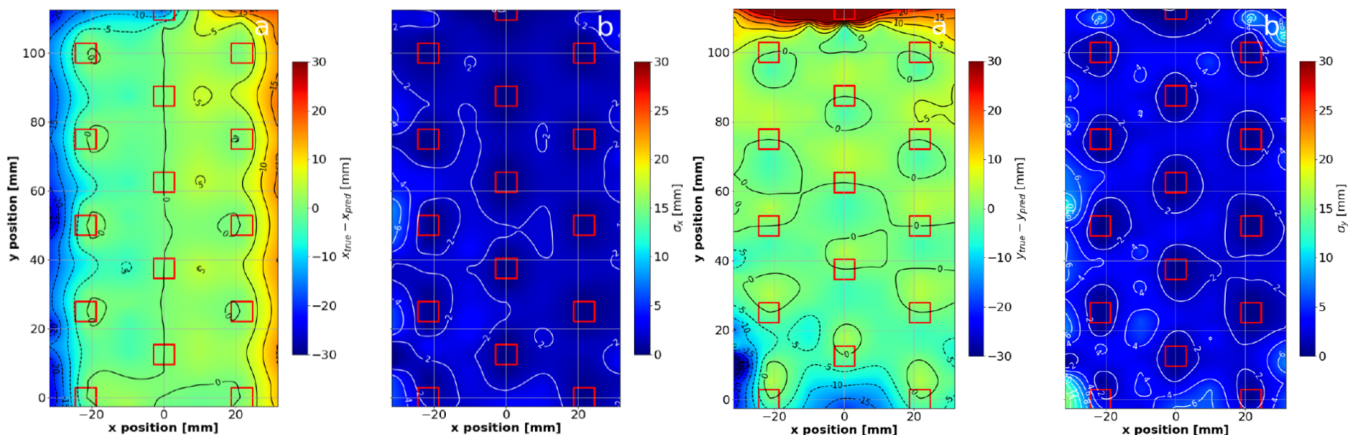

Figure 3: The maps of parameters containing data that allow calibration of the scintillation light readout system, which is the correction of systematic shifts (using maps of $x_{\text {true }}-x_{\text {pred }}$ and $y_{\text {true }}-y_{\text {pred }}$ ) and estimating accuracy of reconstruction (using maps of $\sigma_{x}$ and $\sigma_{y}$ ) [5]. The red line squares represent the SiPM mesh.

with the front-end electronics was embedded in a constant potential cage and mounted inside the decay chamber. Power supply and slow control board were located outside the chamber. The SiPM signals were fed to charge sensitive preamplifiers, converted to time and subsequently digitized by a multihit TDC (CAEN VX1190) with 100 ps resolution. The detector was cooled to remove heat from electronics circuits and to provide optimal condition for SiPM sensors. The concept was tested in the laboratory conditions using alpha particles emitted from ${ }^{241} \mathrm{Am}$ with promising results as shown in Fig 3. The characterisation method of detector performed and described in [5] gave the first results with the average position resolution: $\sigma_{x}=1.87 \mathrm{~mm}$ and $\sigma_{y}=3.07 \mathrm{~mm}$.
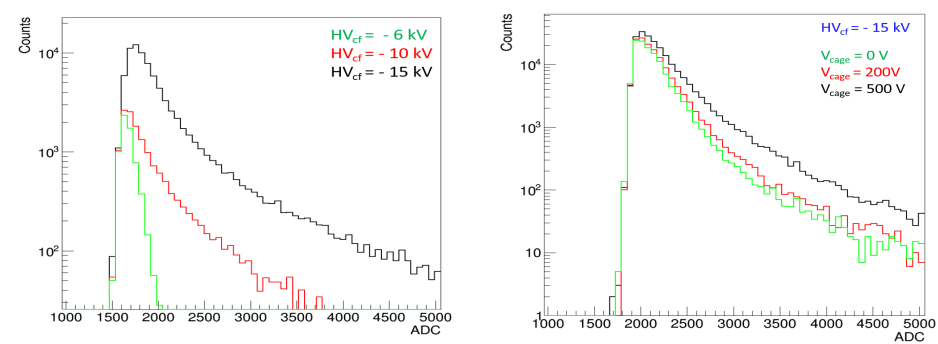

Figure 4: Analysis results of data collected using a cold neutron beam at ILL. Pulse-charge distributions for the different high voltage values on the p-e converter foil and the influence of the potential cage voltage on these distributions, respectively. In case of $\mathrm{HV}_{\mathrm{cf}}=-6 \mathrm{kV}$ distribution is related to the noise.

supported by the National Science Center, Poland under the grant No. UMO-2018/29/B/ST2/02505. successfully used in the test setup on the cold-neutron beam. Figure 4 shows the results obtained for the different high voltage values on the p-e converter foil and the influence of the potential cage voltage on these distribution.

Further optimization of detectors geometry, electronics and used evaluation algorithms is ongoing. This work has been

The presented prototype was

\section{References}

[1] K. Bodek et al., EPJ Web of Conference, 219 (2019) 04001.

[2] M. Perkowski, PhD Thesis, Jagiellonian University and KUL, Krakow/Leuven, 2020.

[3] M. Perkowski et al., Acta Phys. Pol. B, 49 (3) (2018) 261.

[4] S. A. Hoedl, A. R. Young, H. Ade, A. Lozano, Journal of App. Phys. 99, 084904 (2006).

[5] M. Kolodziej, Master Thesis, Jagiellonian University, Krakow 2020. 